\title{
Evaluación del extracto del árbol de neem (Azadirachta indica) como inhibidor de corrosión en medio salino*
}

\author{
\begin{tabular}{|} 
Pedro Meza-Castellar ${ }^{* *}$ \\
Candelaria Tejada-Tovar ${ }^{* * *}$ \\
Shirley Loaiza-Fernández ${ }^{* * * *}$ \\
Alexander Vidales-Manrique $^{* * * * *}$ \\
Ángel Villabona Ortiz
\end{tabular}
}

Recibido: 26/01/2016 - Aceptado: 24/10/2016

DOI: 10.22395/rium.v16n31a1

\section{Resumen}

Se evaluó la eficiencia de inhibición de la corrosión del extracto de las hojas de neem (Azadirachta indica) sobre acero al carbono y aluminio, en contacto por separado con una solución de cloruro de sodio y sulfato de sodio, ambas al 3,5\% p/v. Se determinó la velocidad de corrosión, encontrando que el extracto de neem a menor concentración alcanzó mejores eficiencias sobre acero al carbono en cloruro de sodio con un $78 \%$ de eficiencia usando un 5\% del extracto; en el caso del aluminio los valores de eficiencia alcanzados fueron bajos. Además, mediante el ajuste a la isoterma de Langmuir, se logró determinar que el extracto de neem fue adsorbido espontáneamente en la superficie del acero al carbono y permitió retardar el proceso corrosivo alcanzando eficiencias de inhibición del 90\%. Los resultados de esta investigación permiten establecer que el extracto de neem tiene un alto potencial como inhibidor de la corrosión para ser aplicado en la industria como medio preventivo de esta.

Palabras clave: acero al carbono; aluminio; Azadirachta indica; corrosión; inhibidor.

Artículo derivado del proyecto de investigación Evaluación de la capacidad inhibidora de la corrosión de los extractos del árbol de neem (Azadirachta indica) en medio salino, desarrollada entre 2012 y 2013 con recursos internos de la Universidad de Cartagena (Cartagena, Colombia).

** Magíster en Ingeniería Ambiental. Docente del Programa de Ingeniería de Procesos. Facultad de Ingeniería de la Fundación Universitaria Tecnológico Comfenalco. Sede A: Barrio España, Carrera 44 D \#30A-91, Cartagena, Colombia. pmeza@ tecnocomfenalco.edu.co

**** Magíster en Ingeniería Ambiental. Docente del Programa de Ingeniería Química. Facultad de Ingeniería de la Universidad de Cartagena. Grupo de Investigación en Diseño de procesos y Aprovechamiento de Biomasas (IDAB). Sede Piedra de Bolívar: Avenida El Consulado, Calle 30 \# 48-152, Cartagena, Colombia. ctejadat@unicartagena.edu.co

***** Ingeniera Química. Facultad de Ingeniería de la Universidad de Cartagena. Grupo de Investigación en Diseño de procesos y Aprovechamiento de Biomasas (IDAB). Sede Piedra de Bolívar: Avenida El Consulado, Calle 30 \#48-152, Cartagena, Colombia. shirlyloaiza@gmail.com

****** Ingeniero Químico. Facultad de Ingeniería de la Universidad de Cartagena. Grupo de Investigación en Diseño de procesos y Aprovechamiento de Biomasas (IDAB). Sede Piedra de Bolívar: Avenida El Consulado, Calle 30 \#48-152, Cartagena, Colombia.alevida_78@hotmail.com

******** Magíster en Ingeniería Ambiental. Docente del Programa de Ingeniería Química. Facultad de Ingeniería de la Universidad de Cartagena. Grupo de Investigación en Diseño de procesos y Aprovechamiento de Biomasas (IDAB). Sede Piedra de Bolivar: Avenida El Consulado, Calle 30 \#48-152, Cartagena, Colombia. avillabonao@unicartagena.edu.co 


\title{
Evaluation of extract of neem tree (Azadirachta indica) as inhibitor of corrosion in saline medium
}

\begin{abstract}
The inhibition efficiency of corrosion of the extract of neem leaves (Azadirachta indica) on carbon steel and aluminum was evaluated in contact with a sodium chloride and sodium sulfate solution, both at $3.5 \% \mathrm{p} / \mathrm{v}$. Corrosion speed was determined and it was found that the Neem extract, at a lower concentration, reached better efficiencies on carbon steel in sodium chloride with $78 \%$ efficiency using $5 \%$ of the extract; concerning the aluminum, the efficiency values reached were low. Besides, through the adjustment to the Langmuir's isotherms, it was possible to determine that the nem extract was adsorbed spontaneously on the surface of carbon steel and allowed to delay the corrosive process when reaching inhibition efficiencies of $90 \%$. Results of this research allow establishing that the neem extract has a high potential as corrosion inhibitor to be applied in the industry as corrosion preventive medium.
\end{abstract}

Keywords: carbon steel; aluminum; Azadirachta indica; corrosion; inhibitor.

\section{Avaliação do extrato da árvore de nim (Azadirachta indica) como inibidora de corrosão em meio salino}

\section{Resumo}

Foi avaliada a eficiência de inibição de corrosão do extrato de folha de neem (Azadirachta indica) em aço carbono e alumínio, em contato separadamente com uma solução de cloreto de sódio e sulfato de sódio, ambos com 3,5\% p / v. A taxa de corrosão foi determinada, encontrando que o extrato de neem em menor concentração alcançou melhores eficiências em aço carbono em cloreto de sódio com $78 \%$ de eficiência usando $5 \%$ do extracto; No caso do alumínio, os valores de eficiência alcançados foram baixos. Além disso, ao ajustar-se à isoterma de Langmuir, determinou-se que o extrato de neem foi adsorvido espontaneamente na superfície do aço carbono e permitiu retardar o processo corrosivo atingindo $90 \%$ de eficiências de inibição. Os resultados desta investigação permitem estabelecer que o extrato neem tem um alto potencial como um inibidor de corrosão a ser aplicado na indústria como um meio preventivo deste.

Palavras-chave: aço-carbono; alumínio; Azadirachta indica; corrosão; inibidor. 


\section{INTRODUCCIÓN}

La corrosión se define como el ataque destructivo de un metal por reacción química o electroquímica con el medio ambiente [1].Los costos debidos a este fenómeno son enormes y se estima que corresponde entre el 2 y el $4 \%$ del producto interno bruto de un país industrializado [1-3], y se calcula que al menos el $40 \%$ de las pérdidas pueden evitarse utilizando las tecnologías de prevención disponibles [4]; esta es la razón principal que motiva a investigadores a usar y desarrollar técnicas para la prevención y control de la corrosión como protección catódica, recubrimientos anticorrosivos e inhibidores de corrosión [1], así como la búsqueda de soluciones innovadoras para los impactos ambientales que genera este fenómeno.

Una de las principales medidas de control y prevención de la corrosión es el uso de inhibidores. Los inhibidores son sustancias que al adicionarse en un medio disminuyen la velocidad de corrosión mediante la formación de una capa protectora entre el metal y el medio; los inhibidores pueden ser de origen químico o vegetal [5]. Los más utilizados son los químicos, pero con el pasar de los años esto ha cambiado a nivel local y mundial en la obtención de inhibidores de corrosión a partir de sustancias de origen vegetal provenientes de plantas como Azadirachta indica (neem), Punica granatum (granado), Momordica charantia (melón amargo), y miel natural de Nypa fruticans [6-8]. En el caso de los extractos de hojas y semillas de Phyllanthus amarus, estos fueron evaluados como inhibidores de corrosión en soluciones de ácido sulfúrico y ácido clorhídrico en acero dulce. Otros autores utilizaron unrango de concentraciones de extracto inhibidor con valores entre 0,1 y $2 \mathrm{~g} / \mathrm{L}$; por ende, no se realizó un estudio exhaustivo de la relación de la concentración con la eficiencia en la inhibición de la corrosión [9]. Sin embargo, fue posible identificar responsables de inhibir la corrosión, tales como grasas, proteínas, saponinas, taninos, alcaloides, oxalatos, glucósidos cianogénicos e hidratos de carbono, los cuales son adsorbidos químicamente en la interfaz solución-acero y disminuyen la velocidad con la que el metal se corroe por el contacto que tiene con el medio ácido.

Al estudiar la inhibición de la corrosión de los extractos de la planta Justicia gendarussa en solución de ácido clorhídrico sobre placas de acero, Satapathy y colaboradores demostraron, mediante pruebas de pérdida de peso y técnicas electroquímicas, que la concentración del extracto es directamente proporcional a la eficiencia en la inhibición, alcanzando la mayor eficiencia del 93\% a una concentración de 150 ppm [10].

También, a partir de la planta algodón de seda, o Calotropis procera, se obtuvieron extractos con los cuales se evaluó su posible capacidad inhibidora de la corrosión en hidróxido de sodio sobre placas de acero. En esta investigación se utilizaron diferentes concentraciones de inhibidor y de hidróxido de sodio y se concluyó que con una 
menor concentración de hidróxido de sodio y una alta concentración de inhibidor, se logró disminuir considerablemente la velocidad de corrosión y aumentar la eficiencia inhibidora [11].

En general, estos y otros estudios han demostrado mediante pruebas electroquímicas, de pérdida de peso, entre otros, que presentan una alta eficiencia en la inhibición de la corrosión en acero, aluminio y cobre, tanto en soluciones ácidas como salinas. Además, a diferencia de los inhibidores químicos, no son tóxicos ninocivos para el medio ambiente, se obtienen mediante tecnologías limpias, y no acarrean altos costos[12], principalmente por la gran disponibilidad de estos recursos en muchas regiones del mundo, entre ellas Colombia, cuya biodiversidad cuenta con una flora rica en plantas de diferentes clases y especies; por lo tanto, es importante realizar investigaciones para el aprovechamiento sostenible de la biodiversidad de la región y sus aplicaciones en el sector industrial.

Así en el presente estudio se evalúo la eficiencia del extracto de la hoja del árbol de neem (Azadirachta indica) en la inhibición de la corrosión de acero al carbono y aluminio en soluciones salinas de cloruro de sodio y sulfato de sodio,para lo cual se hizo una caracterización de los extractos mediante pruebas fitoquímicas; posteriormente, por prueba de pérdida de peso, se calculó la velocidad de corrosión en los dos metales.

\section{MATERIALES Y MÉTODOS}

\subsection{Obtención del inhibidor}

Las hojas del árbol de neem (Azadirachta indica) requeridas para la obtención del extracto inhibidor fueron tomadas de la región de los Montes de María del departamento de Bolívar (Colombia); se pesaron250 g de hojas en una balanza electrónica Vibra HT-220E y fueron sometidas a calentamiento en baño maría a $60^{\circ} \mathrm{C}$ en un termostato Water Bath YCW-010E con $600 \mathrm{~mL}$ de agua destilada como solvente, hasta que el volumen se redujo a $180 \mathrm{~mL}$ para garantizar un extracto concentrado,y finalmente se filtró; el procedimiento tardó aproximadamente siete horas.

\subsection{Caracterización del extracto}

Se realizaron pruebas fitoquímicas para identificar la presencia o ausencia de saponinas, taninos, fenoles, grasas y aceites en los extractos inhibidores. Los métodos utilizados para detectar la presencia de cada uno de los constituyentes fitoquímicos en el extracto de neem fueron [13]: extracción Soxhlet para la identificación de aceites y grasas; prueba de la espuma abundante para determinación de saponinas; método gelatina-sal para caracterizar taninos; y fotocolorimetría para identificar fenoles. 


\subsection{Preparación del medio corrosivo}

El medio corrosivo considerado en el presente estudio estuvo conformado por dos soluciones salinas de cloruro de sodio y sulfato de sodio, ambas al 3,5\% p/v, sin agitación, durante los 14 días.

\subsection{Preparación de la solución inhibidora}

Para obtener el extracto inhibidor se pesaron $250 \mathrm{~g}$ de las hojas del árbol de neem en una balanza electrónica Vibra HT-220E y fueron sometidas a un calentamiento en baño maría a $60{ }^{\circ} \mathrm{C}$ en un termostato Water Bath YCW-010E con $600 \mathrm{~mL}$ de agua destilada como solvente hasta que el volumen se redujo a $180 \mathrm{~mL}$ para garantizar un extracto concentrado, y finalmente se filtró.El procedimiento tardó aproximadamente siete horas y se realizó en el Laboratorio de Ingeniería Química de la Universidad de Cartagena (Cartagena, Colombia)

\subsection{Prueba}

Las muestras de acero al carbono fueron láminas de $5 \times 4 \mathrm{~cm}$ con espesor de $3 \mathrm{~mm}$, y para el aluminio se utilizaron láminas de $5 \times 4 \mathrm{~cm}$ con espesor de $1 \mathrm{~mm}$.

Los experimentos fueron llevados a cabo a temperatura ambiente exponiendo las muestras metálicas a dos soluciones salinas: una de cloruro de sodio y otra de sulfato de sodio, ambas al 3,5\% p/v, teniendo en cuenta que aproximadamente a ese porcentaje están presentes los cloruros y sulfatos de sodio en el agua de mar [14]. Este experimento se realizó lavando todas las placas con etanol 95\% v/v para remover cualquier impureza en la superficie; posteriormente se secaron con un paño de seda a temperatura ambiente durante $1 \mathrm{~min}$, y se pesaron para conocer su masa antes de que se presentara la corrosión, sin ningún pre-tratamiento. Cada cupón fue introducido en un recipiente con aproximadamente $180 \mathrm{~mL}$ de las soluciones salinas por separado con las dos concentraciones de extractos; la inmersión se llevó a cabo durante 14 días sin agitación[15]. Las evaluaciones de inhibición de la corrosión se realizaron cada 24 horas; las placas eran retiradas para pesarlas y ser sumergidas de nuevo en la correspondiente solución. Los datos experimentales de pérdida de peso del metal en gramos y tiempo de inmersión en el medio corrosivo en horas se graficaron para obtener la pendiente que corresponde a la velocidad de corrosión para cada prueba.

En la tabla 1, se observan los experimentos realizados para la prueba de pérdida de peso del acero al carbono y al aluminio. Cabe destacar que se realizó una réplica del experimento para verificar los datos obtenidos y calcular una estimación más precisa del efecto de cada factor en el estudio. 
20 P. Meza-Castellar - C. Tejada-Tovar - S. Loaiza-Fernández - A. Vidales-Manrique - A. Villabona Ortiz

Tabla 1. Experimentos realizados para determinar la pérdida de peso.

\begin{tabular}{|c|c|l|l|}
\hline $\begin{array}{c}\text { Número de } \\
\text { experimento }\end{array}$ & $\begin{array}{c}\text { Concentración } \\
\text { del inhibidor } \%\end{array}$ & \multicolumn{1}{|c|}{ Metal } & \multicolumn{1}{c|}{ Sal } \\
\hline 1 & 5 & Aluminio & Cloruro de sodio $(\mathrm{NaCl})$ \\
\hline 2 & 5 & Aluminio & Sulfato de sodio $\left(\mathrm{Na}_{2} \mathrm{SO}_{4}\right)$ \\
\hline 3 & 5 & Acero al carbono & Cloruro de sodio $(\mathrm{NaCl})$ \\
\hline 4 & 5 & Acero al carbono & Sulfato de sodio $\left(\mathrm{Na}_{2} \mathrm{SO}_{4}\right)$ \\
\hline 5 & 10 & Aluminio & Cloruro de sodio $(\mathrm{NaCl})$ \\
\hline 6 & 10 & Aluminio & Sulfato de sodio $\left(\mathrm{Na}_{2} \mathrm{SO} 4\right)$ \\
\hline 7 & 10 & Acero al carbono & Cloruro de sodio $(\mathrm{NaCl})$ \\
\hline 8 & 10 & Acero al carbono & Sulfato de sodio $\left(\mathrm{Na}_{2} \mathrm{SO} 4\right)$ \\
\hline
\end{tabular}

\subsection{Cálculo de la eficiencia}

La eficiencia de los inhibidores se define como la capacidad para inhibir la corrosión del metal, y se establece en función de la velocidad de corrosión del metal sin inhibidor. Una vez se obtuvieron las velocidades de corrosión se calculó la eficiencia inhibidora (EI) del extracto de neem utilizando la ecuación 1:

$$
E I(\%)=\frac{v c-v c_{\text {inhibidor }}}{v c} \times 100
$$

Donde $v c$, es la velocidad de corrosión sin inhibidor y $v c_{\text {inhibidor }}$ corresponde al sistema al que se le adicionó el extracto de neem.

Otro parámetro que se tuvo en cuenta para el análisis de los resultados fue el grado de cobertura de la superficie $\theta$, el cual representa la parte del metal cubierta por las moléculas del inhibidor y se calculó mediante la siguiente ecuación 2 [17]:

$$
\theta=\frac{E I(\%)}{100}
$$

Además, para determinar la interacción entre el metal y el extracto inhibidor, se calculóla isoterma de adsorción realizando seis experimentos a diferentes concentraciones del extracto $(0,5 \%, 1 \%, 3 \%, 5 \%$ y $7 \%$ ) y uno sin inhibidor, considerado como blanco. El procedimiento utilizado fue el mismo empleado en la prueba de pérdida de peso, con un tiempo de inmersión de 24 horas; las placas fueron retiradas, lavadas y secadas cada 12 horas. 


\section{RESULTADOS Y DISCUSIÓN}

\subsection{Caracterización fitoquímica del extracto}

En la tabla 2 se observan los resultados de la caracterización fitoquímica del extracto de Neem (Azadirachta Indica).Los taninos y fenoles son compuestos orgánicos con fórmulas moleculares $\mathrm{C}_{14} \mathrm{H}_{14} \mathrm{O}_{11}$ y $\mathrm{C}_{6} \mathrm{H}_{6} \mathrm{O}$, respectivamente, que gracias a sus átomos de oxígeno son adsorbidos sobre la superficie del metal formando complejos que actúan como una capa protectora, disminuyendo el área de contacto entre el metal y el medio corrosivo y, por consiguiente, la velocidad de corrosión. La presencia de grupos $\mathrm{OH}$ en los taninos y fenoles les confirió la capacidad de formar quelatos y sales con iones férricos y otros cationes metálicos [17-18]; los complejos formados causaron la obstrucción de micro-ánodos, que se generan en la superficie del metal cuando entran en contacto con un electrolito o medio corrosivo; por lo tanto, permitieron retardar la disolución del metal [19].

Tabla 2. Constituyentes fitoquímicos del extracto de Neem

\begin{tabular}{|l|l|}
\hline \multicolumn{1}{|c|}{ Componente } & \multicolumn{1}{c|}{ Resultado } \\
\hline Aceites y grasas & No detectable \\
\hline Saponinas & No detectable \\
\hline Taninos & $0,143 \%$ \\
\hline Fenoles & $0,08 \%$ \\
\hline
\end{tabular}

Fuente: elaboración propia.

\subsection{Prueba de pérdida de peso}

En la figura 1 se presentan las gráficas de pérdida de peso de acero al carbono versus tiempo de inmersión de las placas en presencia y ausencia (blanco) del extracto inhibidor.

La figura 1 (promedio de los datos de experimento y réplica para el acero al carbono) revela que la pérdida de peso de las placas de acero al carbono aumenta con el tiempo de inmersión a lo largo de un período de 336horas, y es mayor en la solución de sulfato de sodio que en cloruro de sodio, lo cual se asoció al efecto de la fuerza iónica del sulfato de sodio, que equivale a tres veces su concentración debido a la presencia de dos iones sodio y un ion sulfato en su estructura, en comparación con el cloruro de sodio, que equivale a una vez su concentración, dada la presencia de un ion sodio y un ion cloruro[20]. Lo anterior permitió que las cargas eléctricasviajaran con 
mayor facilidad del ánodo al cátodo en la solución de sulfato de sodio favoreciendo el proceso corrosivo.

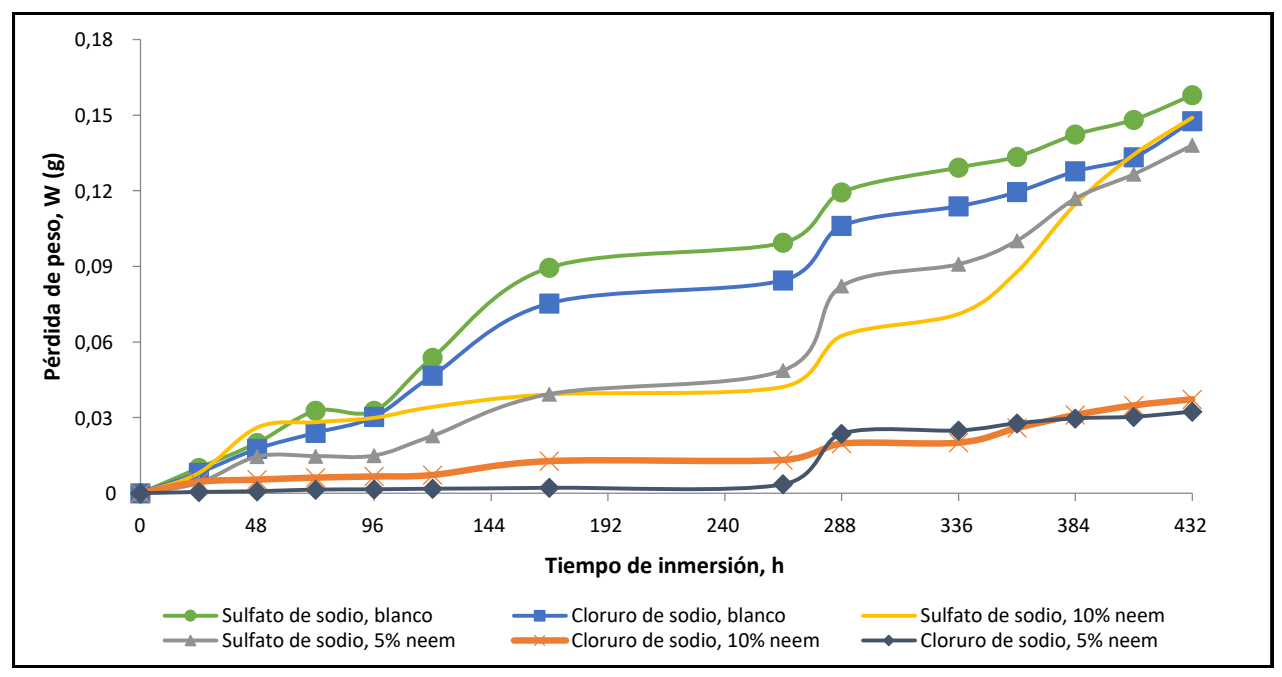

Figura 1. Curvas de pérdida de peso vs. Tiempo de inmersión para el acero al carbono en cloruro de sodio y sulfato de sodio.

Fuente: elaboración propia.

De igual manera, la pérdida de peso aumentó con la concentración del extracto inhibidor lo cual indicó que para la concentración utilizada de $10 \%$, el extracto ha perdido su capacidad inhibidora debido a que acelera el proceso corrosivo sobre el metal por lo que se obtuvieron mayores velocidades de corrosión que cuando se evaluó la concentración del 5\%; para el caso del acero al carbono de $0,0859 \mathrm{mg} /$ hcon una concentración de extracto inhibidor del $10 \%$ frente a $0,0747 \mathrm{mg} / \mathrm{hpara}$ el $5 \%$, y en las placas de aluminio, los valores fueron de $0,3440 \mathrm{mg} / \mathrm{hy} 0,3188 \mathrm{mg} / \mathrm{h}$, respectivamente.

Se observó,además, que las curvas de pérdida de peso para los experimentos en blanco (ausencia de extracto) tienen una mayor pendiente que las curvas de la solución de sulfato de sodio y, a su vez, que las curvas para la solución de cloruro de sodio, lo que significa que el inhibidor funcionó en ambas soluciones siendo más visible el efecto inhibidor encloruro de sodio al retardarel proceso de corrosión durante el ensayo y la réplica [21].

En la figura 2, se presentan las curvas de pérdida de peso de aluminio versus tiempo de inmersión de las placas en presencia y ausencia (blanco) del extracto inhibidor. 


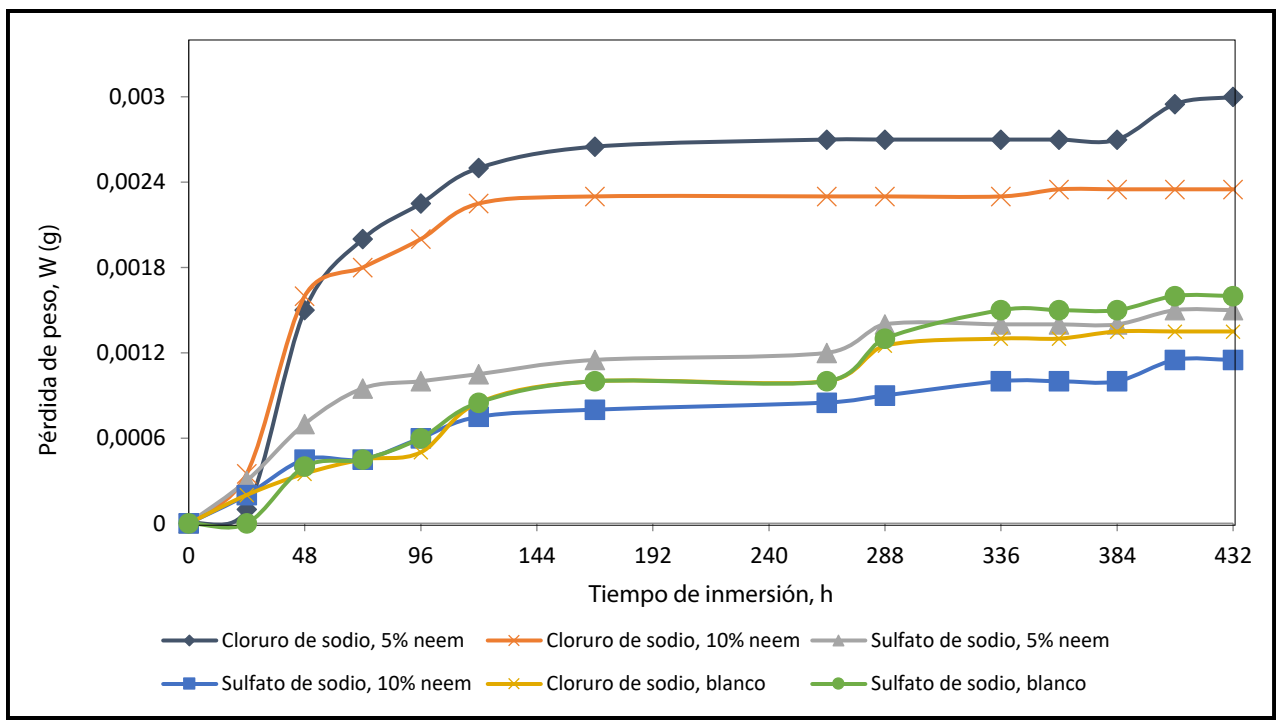

Figura 2. Curvas de pérdida de peso vs. Tiempo de inmersión para el aluminio en cloruro de sodio y sulfato de sodio.

Fuente: elaboración propia.

Una inspección de las curvas de pérdida de peso de la figura 2 reveló que la pérdida de peso aumentó a lo largo de las 432 horas que duraron los experimentos y fuemayor para las placas sumergidas en cloruro de sodio $3,5 \% \mathrm{p} / \mathrm{v}$ que para las pruebas en blanco y en presencia de sulfato de sodio, debido a que al entrar en contacto las placas con el sulfato de sodio, el aluminio, mediante una reacción anódica a partir de la cual se desarrolló la corrosión, se ionizó y quedó cargado con un exceso de cargas positivas facilitando una mayor conducción de energía y una reacción más rápida con los taninos del extracto,que favoreció la formación de quelatos y de una capa protectora que disminuyó el área de contacto entre el medio corrosivo y el metal, reduciendo asíla pérdida de peso [22-23]. Para el caso del cloruro de sodio con aluminio, el ion aluminio reaccionó con la sal y formó una capa de cloruro de aluminio u oxicloruro de aluminio, pero después de un tiempoeste se hidrolizó formando hidróxido de aluminio,ocasionando, de acuerdo con Balbo y colaboradores, unadisminuciónen el pH de la solución, pasando de $\mathrm{pH}$ básico a pH ácido, lo que favoreció la presencia de iones hidronio y, por tanto, la velocidad de corrosión aumentó [24]. A partir de estos valores se calculó la eficiencia en la inhibición de la corrosión utilizando la ecuación 1 y el grado de recubrimiento del metal con la ecuación 2. Los datos obtenidos se presentan en la tabla 3. 
24 P. Meza-Castellar - C. Tejada-Tovar - S. Loaiza-Fernández - A. Vidales-Manrique - A. Villabona Ortiz

Tabla 3. Comparativo de eficiencias de inhibición del extracto de Neem.

\begin{tabular}{|c|c|c|c|c|c|}
\hline \multirow{2}{*}{$\begin{array}{c}\text { Medio } \\
\text { corrosivo }\end{array}$} & \multirow{2}{*}{$\begin{array}{l}\text { Concen- } \\
\text { tración del } \\
\text { extracto }\end{array}$} & \multicolumn{2}{|c|}{ Acero al carbono } & \multicolumn{2}{|c|}{ Aluminio } \\
\hline & & $\begin{array}{c}\text { Eficiencia } \\
\text { inhibidora }(\%)\end{array}$ & $\begin{array}{c}\text { Grado de } \\
\text { recubrimiento }\end{array}$ & $\begin{array}{c}\text { Eficiencia } \\
\text { inhibidora (\%) }\end{array}$ & $\begin{array}{c}\text { Grado de } \\
\text { recubrimiento }\end{array}$ \\
\hline \multirow{2}{*}{$\begin{array}{l}\text { Cloruro } \\
\text { de sodio }\end{array}$} & $5 \%$ & 78,076 & 0,780776 & $-121,2733$ & $-1,212733$ \\
\hline & $10 \%$ & 74,788 & 0,74788 & $-72,6708$ & $-0,726708$ \\
\hline \multirow{2}{*}{$\begin{array}{l}\text { Sulfato de } \\
\text { sodio }\end{array}$} & $5 \%$ & 13,626 & 0,13626 & 6,6754 & 0,066754 \\
\hline & $10 \%$ & 6,804 & 0,06804 & 28,3377 & 0,283377 \\
\hline
\end{tabular}

Fuente: elaboración propia.

El extracto de neem presentó mayores eficiencias de inhibición de la corrosión sobre placas de acero al carbono que de aluminio, debido a que los iones férricos y ferrosos que libera el acero reaccionan con los taninos del extracto y se forman quelatos bastante estables que actúan como una capa protectora que impideel contacto entre el electrolito y el metal, evitando entonces que el hierro se siga solubilizando; como se observa en la tabla 4, las eficiencias en la inhibición de la corrosión (EI) para el acero al carbono evaluado en cloruro de sodio son bastante altas con un porcentaje máximo de $78,076 \%$ y, por el contrario, para el sulfato de sodio las eficiencias no superan el 13,626\%. Además, a diferencia del acero al carbono que presentó una corrosión homogénea, en el aluminio se desarrolló una actividad corrosiva denominada picadura que se define como la disolución localizada y acelerada de un metal; se produjo porque el aluminio es un metal auto-pasivante, es decir, crea de manera espontánea una película pasivada de óxido de aluminio la cual inicialmente reduce la velocidad de corrosión, pero al transcurrir algún tiempo y por presencia de los iones cloruro y sulfato, la película sufrió rupturas localizadas, lo que aceleró la disolución del metal y la velocidad de corrosión.

Tabla 4. Análisis de Varianza para la eficiencia inhibidora.

\begin{tabular}{|l|c|}
\hline \multicolumn{1}{|c|}{ Fuente } & Valor- $p$ \\
\hline A: concentracion inhibidor & 0,7014 \\
\hline B: metal & 0,0000 \\
\hline C: sal & 0,0002 \\
\hline AB & 0,1088 \\
\hline AC & 0,3913 \\
\hline BC & 0,0000 \\
\hline
\end{tabular}

Fuente: elaboración propia. 


\subsection{Análisis de varianza ANOVA}

Los factores y corridas del diseño experimental se observan en la tabla 5:

Tabla 5. Diseño experimental para análisis de varianza ANOVA.

\begin{tabular}{|c|c|c|c|c|}
\hline \multicolumn{4}{|c|}{ Factores } & Variable respuesta \\
\hline Prueba & $\begin{array}{l}\text { Concentración } \\
\text { inhibidor }\end{array}$ & Metal & Sal & $\begin{array}{c}\text { Eficiencia de inhibición } \\
(\%)\end{array}$ \\
\hline 1 & $5 \%$ & Aluminio & $\mathrm{NaCl}$ & $-121,2733$ \\
\hline 2 & $5 \%$ & Aluminio & $\mathrm{Na}_{2} \mathrm{SO}_{4}$ & 6,6754 \\
\hline 3 & $5 \%$ & Acero al carbono & $\mathrm{NaCl}$ & 78,076 \\
\hline 4 & $5 \%$ & Acero al carbono & $\mathrm{Na}_{2} \mathrm{SO}_{4}$ & 13,626 \\
\hline 5 & $10 \%$ & Aluminio & $\mathrm{NaCl}$ & $-72,6708$ \\
\hline 6 & $10 \%$ & Aluminio & $\mathrm{Na}_{2} \mathrm{SO}_{4}$ & 28,3377 \\
\hline 7 & $10 \%$ & Acero al carbono & $\mathrm{NaCl}$ & 74,788 \\
\hline 8 & $10 \%$ & Acero al carbono & $\mathrm{Na}_{2} \mathrm{SO}_{4}$ & 6,804 \\
\hline
\end{tabular}

Fuente: elaboración propia.

Mediante el análisis se determinó que los factores tipo de metal, tipo de sal y la interacción entre estos tuvieron una incidencia significativa sobre la variable de respuesta, ya que tienen un valor-p menor a 0,005 (tabla 5).

\subsection{Isoterma de adsorción}

El estudio de las isotermas se realizó con la condición que mejor resultado arrojó (concentración 5\%; acero al carbono y $\mathrm{NaCl}$ ). Los datos obtenidos de las pruebas de isoterma de adsorción a $25^{\circ} \mathrm{C}$ presentaron un ajuste a la isoterma de Langmuir, y permitieron conocer la interacción que se presentó entre el extracto inhibidor y el metal (acero al carbono), es decir, la manera como el inhibidor es adsorbido en la superficie del acero, mediante el cálculo de la energía libre de Gibbs [22]. Al graficar la relación entre la concentración del inhibidor y la superficie cubierta contrala concentración del inhibidor, se obtuvo la figura 3 , que presentó un ajuste bastante cercano a una línea recta $\left(\mathrm{R}^{2}=0,9967\right)$. 


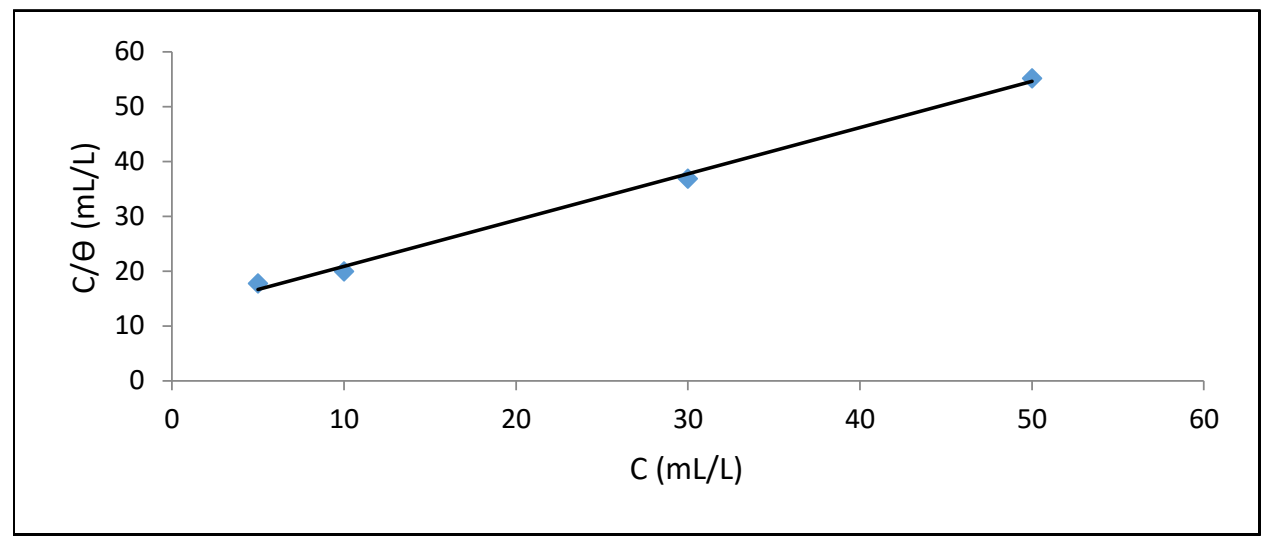

Figura 3. Isoterma de adsorción de Langmuir. (C/ $\theta$ v C).

Fuente: elaboración propia.

La ecuación para la isoterma de Langmuir, en función de los parámetros estudiados es:

$$
\frac{C}{\theta}=\frac{1}{K}+C
$$

C: concentración del extracto inhibidor $(\mathrm{mL} / \mathrm{L})$;

$\theta$ : superficie cubierta;

$\mathrm{K}$ : constante de adsorción $(\mathrm{L} / \mathrm{mL})[25]$.

Al comparar una ecuación lineal con la ecuación resultante de la regresión de los datos de la figura 3 , se obtuvo que, donde $1 / \mathrm{K}$ es el intercepto con el eje " $\mathrm{y}$ ", entonces la constante de adsorción es:, donde

Así, mediante la siguiente ecuación se calculó la energía libre de Gibbs [26]:

$$
\Delta G=-R T \ln \left[K \cdot C_{A P}\right]
$$

Donde:

Donde: R: Constante universal de los gases ( $\mathrm{J} / \mathrm{mol} \mathrm{K})$; T: Temperatura ambiente; $\mathrm{K}$ : Constante de adsorción $(\mathrm{L} / \mathrm{mL}) ; \mathrm{C}_{\mathrm{AP}}$ : Concentración del agua pura la cual equivale a $1000 \mathrm{~mL} / \mathrm{L}$ o $1000 \mathrm{~g} / \mathrm{L}$ o $55,55 \mathrm{~mol} / \mathrm{L}$. 


$$
\begin{gathered}
\Delta G=-\left(8,314 \frac{J}{\mathrm{~mol} \cdot K}\right)(298 K) \ln \left[0,0804829 \frac{\mathrm{L}}{\mathrm{mL}} \cdot 1000 \frac{\mathrm{mL}}{\mathrm{L}}\right]=-10871,6967 \frac{\mathrm{J}}{\mathrm{mol}} \\
\Delta G=-10,87 \frac{\mathrm{KJ}}{\mathrm{mol}}
\end{gathered}
$$

En general, la adsorción de inhibidores de corrosión sobre metales puede prevenir las reacciones anódicas, catódicas o ambas, responsables de la pila que origina corrosión. Este proceso puede ser físico, químico o una combinación de ambos.

De acuerdo con estudios realizados utilizando diferentes extractos vegetales como inhibidores de corrosión evaluados sobre metales se estableció que valores negativos para la energía libre de Gibbs hasta $-20 \mathrm{~kJ} / \mathrm{mol}$ corresponden al mecanismo de fisisorción e indican la espontaneidad del proceso; valores de $-40 \mathrm{~kJ} / \mathrm{mol}$ o más negativos son coherentes con quimisorción[26]. En las investigaciones, al determinar el mecanismo de adsorción metal-extracto, se ha encontrado que la mayoría de las interacciones que se presentan son por fisisorción; el extracto de Euphorbia falcatafue evaluado en placas de acero al carbono y al calcular la energía libre de Gibbs se obtuvo un valor de $-23,92 \mathrm{~kJ} \mathrm{~mol}^{-1}$ [27]. Otros autores alcanzaron valores similares de -20,1 kJ/moly -19,9 $\mathrm{kJ} /$ molpara $45^{\circ} \mathrm{C} \mathrm{y} 25^{\circ} \mathrm{C}$, respectivamente al estudiar la capacidad de inhibición de la corrosión de las hojas del árbol Osmanthus fragans sobre acero al carbono en ácido clorhídrico 1M [28].Al estudiar el comportamiento de adsorción de la Uncariagambir sobre acero al carbono en ácido clorhídrico la energía libre de Gibbs alcanzó un valor de $-21,957 \mathrm{~kJ} / \mathrm{mol}[29]$.

Con base en lo anterior y en el valor calculado para la energía libre de Gibbs de -10,87kJ/mol se concluyó que el mecanismo de adsorción del presente estudio corresponde a adsorción física o fisisorción lo que implicó la rápida interacción entre adsorbente y adsorbato. Sin embargo, la adsorción física disminuye fácilmente de la superficie con el aumento de la temperatura debido a que las moléculas del inhibidor se mantienen unidas a la superficie del metal por medio de fuerzas de Van der Waals, las cuales son relativamente débiles al compararlas con enlaces químicos covalentes e iónicos [30].

Los resultados de la prueba de pérdida de peso permitieron concluir que de las concentraciones del extracto utilizadas inicialmente (5 y 10\%), se logró una máxima eficiencia inhibidora del 78,076\% a menor concentración, y al aumentar esta, se aceleró el proceso corrosivo debido a que ocurrió lo mismo con la velocidad de corrosión; esto conlleva una menor eficiencia $(74,788 \%)$, por lo cual en este punto se evaluó un rango más amplio de concentraciones de extracto $(0,5 ; 1: 3 ; 5 \mathrm{y} 7 \%)$ para establecer desde y hasta qué concentración es viable utilizar el inhibidor obtenido de las hojas del árbol de neem. 
En la tabla 6, se observan las velocidades de corrosión en cloruro de sodio 3,5\% $\mathrm{p} / \mathrm{v}$ y la eficiencia inhibidora para cada una de las concentraciones mencionadas. Los resultados indicaron claramente que la menor velocidad de corrosión y la mayor eficiencia inhibidora se lograron con una concentración del 5\% v/v. De igual manera, a medida que disminuye la concentración sucede lo mismo con la eficiencia. El extracto inhibidor tiene una máxima concentración permisible a utilizar del $5 \%$ por lo cual al usar valores superiores, se da un aumento en la velocidad de corrosión y este pierde su capacidad inhibidora [31].

Tabla 6. Velocidad de corrosión en cloruro de sodio 3,5\% p/vy eficiencia de inhibición a diferentes concentraciones de extracto inhibidor.

\begin{tabular}{|c|c|c|}
\hline $\begin{array}{c}\text { Concentración } \\
\text { extracto }\end{array}$ & $\begin{array}{c}\text { Velocidad de } \\
\text { corrosión } \\
(M g / h)\end{array}$ & $\begin{array}{c}\text { Eficiencia inhibidora } \\
(\%)\end{array}$ \\
\hline $7 \%$ & 0,19583 & $-46,875$ \\
\hline $5 \%$ & 0,0125 & 90,625 \\
\hline $3 \%$ & 0,0250 & 81,25 \\
\hline $1 \%$ & 0,0667 & 50 \\
\hline $0.5 \%$ & 0,0958 & 28,125 \\
\hline Blanco & 0,1333 & - \\
\hline
\end{tabular}

Fuente: elaboración propia.

\section{CONCLUSIONES}

En el análisis de pérdida de peso se alcanzó una máxima eficiencia de la inhibición de la corrosión del acero al carbono en cloruro de sodio del 78,076\% porque el hierro reacciona con los taninos creando una capa protectora que retrasa el proceso corrosivo. Al evaluarla en sulfato de sodio, se obtuvo una menor eficiencia porque el hierro reacciona con el ion sulfato, y los taninos no reaccionan, comportándose como electrolitos aumentando la velocidad de corrosión y disminuyendo considerablemente la eficiencia. El extracto de neem no funciona como inhibidor de la corrosión en las placas de aluminio debido a que este metal presenta corrosión por agujeros que forma una película protectora bastante débil la cual, que al entrar en contacto con iones cloruro y sulfato de los medios corrosivos utilizados, sufre rupturas localizadas, acelerando la disolución del metal y la velocidad de corrosión. Mediante el ajuste a la isoterma de adsorción de Langmuir se calculó un valor para la energía libre de Gibbs de -10,87 kJ/ mol, el cual corresponde a una adsorción física, lo que implicó la rápida interacción entre adsorbente y adsorbato, es decir, el extracto inhibidor de neem fue adsorbido 
espontáneamente en la superficie del acero al carbono y se retardó el proceso corrosivo, lo que permitió alcanzar buenas eficiencias de inhibición en el rango de 50 a $90 \%$ en las placas de metal sumergidas en cloruro de sodio.

\section{REFERENCIAS}

[1] H. Uhlig y R. Revie. Corrosion and corrosion control: an introduction to corrosion, en Science and engineering, 4a ed., New Jersey: John Wiley \& Sons, Inc., Publication, 2008, $513 \mathrm{p}$.

[2] N. Huynh. "The inhibition of copper corrosion in aqueous environments heterocyclic compounds". Tesis de doctorado ÁREA. Universidad Tecnológica de Queensland, 2004, 99 p.

[3] S. Ahmad. "Reinforcement corrosion in concrete structures, its monitoring and service life prediction--a review". Cement and Concrete Composites, Vol. 25, pp. 459-471, 2003.

[4] L. Godínez, Y. Meas, R. Ortega-Borges y A. Corona. "Los inhibidores de corrosión". Revista de Metalurgia, Vol. 39, N. 2, pp. 140-158, 2003.

[5] V. S. Sastri, (2011). "Green CorrosionInhibitors: Theory and Practice”. Canada: John Wiley \& Sons, Inc., Publication, 2011, 328 p.

[6] M. Quraishi, I. Faroogi y P. Saini. "Investigation of Some Green Compounds as Corrosion and Scale Inhibitors for Cooling Systems”.Journal Corrosion, Vol. 55, pp. 493-496, 1999.

[7] A. El-Etre y M. Abdallah."Natural honey as corrosion inhibitor for metals and alloys". II. C-steel in high saline water. Corrosion Science, Vol. 42, pp. 731-733, 2000.

[8] K. Orubite y N. Oforka. "Inhibition of the corrosion of mild steel in hydrochloric acid solutions by the extracts of leaves of Nypa fruticans Wurmb".Materials Letters, Vol. 58, pp. 1768-1772, 2004.

[9] P. Okafor, M. Ikpi, E. Ebemso, U. Ekpe y S. Umoren."Inhibitory action of Phyllanthusamarus extracts on the corrosion of mild steel in acidic media". CorrosionScience, Vol. 50, pp. 2310-2317, 2008.

[10] A. Satapathy, G. Gunasekaran, S. Sahoo, A. Kumar y P.Rodrigues. "Corrosion inhibition by Justiciagendarussa plant extract in hydrochloric acid solution”. Corrosion Science, Vol. 51, pp. 2848-2856, 2009.

[11] S. Kumar, S. Arora, M. Sharma, P. Arora y S. Mathur. "Synergistic effect of Calotropis plant in controlling corrosion of mild steel in basic solution".Journal of the Chilean Chemical Society, Vol. 54, pp. 83-88, 2009.

[12] D. Prabhu, y P.Rao. Coriandrumsativum L. - A novel green inhibitor for the corrosion inhibition of aluminium in $1.0 \mathrm{M}$ phosphoric acid solution. Journal of Environmental Chemical Engineering, 1, pp. 676-683, 2013.

[13] M. Berrocal, E. Altamiranda. "Estudio de la eficiencia de inhibidores de corrosión a partir de extractos vegetales". Tesis de pregrado. Universidad de Cartagena, 2013, 101 p. 
[14] J. Cifuentes, M. Torres y M. Frías. El océano y sus recursos II. Las ciencias del mar: oceanografía geológica y oceanografía química, 3a ed., México: Editorial Fondo de Cultura Económica, SEP, CONACyT, 1986, 170 p.

[15] S.Garai,S.Garai, P.Jaisankar, J.K. Singh, y A. Elango. A comprehensive study on crude methanolic extract of Artemisia pallens (Asteraceae) and its active component as effective corrosion inhibitors of mild steel in acid solution. Corrosion Science, Vol. 60, pp. 193-204, 2012.

[16] O. Abiola, E. Odin, D. Olowoyo y T.Adeloye. “Gossipiumhirsutuml. Extract as green corrosion inhibitor for Aluminum in $\mathrm{HCl}$ solution”. Bulletin of the Chemical Society of Ethiopia, Vol. 45, pp. 475-480, 2011.

[17] R. Silveira, E. Cassel y D. Schermann. "Black Wattle Tannin as Steel Corrosion Inhibitor". ISRN Corrosion, Vol. 2012, pp. 1-9, 2012.

[18] A. Nahl'e, I. Abu-Abdoun, I. Abdel-Rahman, y M. Al-Khayat. "UAE Neem Extract as a Corrosion Inhibitor for Carbon Steel in $\mathrm{HCl}$ Solution". International Journal of Corrosion, Vol. 2010, pp. 1-9, 2010.

[19] B. Amitha y J. Bharathi.“Green Inhibitors for Corrosion Protection of Metals and Alloys: An Overview”. International Journal of Corrosion, Vol. 2012, pp. 1-15, 2012.

[20] D. Skoog, D. West, F. James Holler y S. Crouch. Fundamentos de Química Analítica. 8va ed., México: S.A. Ediciones Paraninfo, 2005, 1196 p.

[21] A. El-Etre. "Inhibition of aluminum corrosion using Opuntia extract". Corrosion Science. Vol. 45, pp. 2485-2495, 2003.

[22] Z. Dan, I.Mutob, y N. Harab. "Effects of environmental factors on atmospheric corrosion of aluminium and its alloys under constant dew point conditions". Corrosion Science, vol. 57, pp. 22-29, 2012.

[23] J. Ávila y J. Genescá. Más allá de la herrumbre I. 1a ed., México: Fondo De Cultura Económica, S. A. de C.V., 1987, pp. 50-85.

[24] A. Balbo, A. Frignani, V. Grassi y F.Zucchi."Corrosion inhibition by anionic surfactants of AA2198 Li-containingaluminium alloy in chloride solutions”. Corrosion Science, Vol. 73, pp. 80-88, 2013.

[25] R. Touri, M. Cenoui, M. Bakri y M. Touhami.“Sodium gluconate as corrosion and scale inhibitor of ordinary steel in simulated cooling water".Corrosion Science, Vol. 50, pp.15301537, 2008.

[26] G. Quartarone, L. Ronchin, A. Vavasori, C. Tortato y L. Bonaldo. "Inhibitive action of gramine towards corrosion of mild Steel in deaerated 1.0 M hydrochloric acid solutions". Corrosion Science, Vol. 64, pp. 82-89, 2012.

[27] A. Bribria, M. Tabyaouia, B. Tabyaouia, H. Attaric y F. Bentissc. "The use of Euphorbia falcata extract as eco-friendly corrosion inhibitor of carbon steel in hydrochloric acid solution”. Materials Chemistry and Physics, Vol. 141, pp. 240-247, 2013. 
[28] L. Lia, X. Zhanga, J. Leia, J. Hea, S. Zhanga y F. Panb. "Adsorption and corrosion inhibition of Osmanthus fragran leaves extract on carbon steel”. Corrosion Science, Vol. 63, pp. 82-90, 2012.

[29] M. Hussin y M. Kassim."The corrosion inhibition and adsorption behavior of Uncariagambirextract on mild steel in $1 \mathrm{M} \mathrm{HCl".} \mathrm{Materials} \mathrm{Chemistry} \mathrm{and} \mathrm{Physics,} \mathrm{Vol.} \mathrm{125,} \mathrm{pp.} \mathrm{461-468,}$ 2011.

[30] J. Condon. Surface Area and Porosity Determinations by Physisorption: Measurements and Theory. 1a Ed., Harriman: Elsevier, 2006, pp. 2-10.

[31] G. Pinder y W. Gray. Essentials of Multiphase Flow in Porous Media. 1a ed., New Jersey: John Wiley \& Sons, Inc., Publication, 2008, pp. 180-186. 